\title{
In-vitro activity of $\beta$-lactams/trimethoprim-sulfamethoxazole combinations against different strains of Burkholderia pseudomallei
}

\author{
Mohamad, N.I. ${ }^{1,2}$, Harun, A. ${ }^{1,3}$, Hasan, H. ${ }^{1,3}$, Deris, Z.Z.1,3* \\ ${ }^{1}$ Department of Medical Microbiology and Parasitology, School of Medical Sciences, Universiti Sains Malaysia, Health Campus, 16150, Kubang Kerian, \\ Kelantan, Malaysia \\ ${ }^{2}$ School of Biology, Faculty of Applied Sciences, Universiti Teknologi MARA, 40450 Shah Alam, Selangor, Malaysia \\ ${ }^{3}$ Hospital Universiti Sains Malaysia, 16150, Kubang Kerian, Kelantan, Malaysia \\ *Corresponding author: zakuan@usm.my
}

\section{ARTICLE HISTORY}

Received: 18 August 2021

Revised: 9 December 2021

Accepted: 9 December 2021

Published: 20 February 2022

\begin{abstract}
Trimethoprim-sulfamethoxazole is an active agent against Burkholderia pseudomallei and is being used in intensive and maintenance phases of melioidosis therapy. In this study, we evaluated the bactericidal activities of $\beta$-lactams (imipenem, ceftazidime and amoxicillinclavulanate) alone and in combinations with trimethoprim-sulfamethoxazole against B. pseudomallei. Four clinical strains of B. pseudomallei were selected based on different genotypes that are frequently found in Malaysia. The minimum inhibitory concentrations of trimethoprim-sulfamethoxazole, ceftazidime, imipenem and amoxicillin-clavulanate were determined using microdilution broth method. The bactericidal activities and synergy effects of $\beta$-lactams and/or trimethoprim-sulfamethoxazole were evaluated by checkerboard and static time-kill analyses at $1 \times$ MIC concentration of each antibiotic. Using checkerboard method, the $\beta$-lactam/trimethoprim-sulfamethoxazole combinations exhibited $\Sigma$ FIC of 0.75-4.00. In time-kill analysis, ceftazidime/trimethoprim-sulfamethoxazole combination demonstrated synergy against three strains (less 2.25-2.41 $\log _{10} \mathrm{CFU} / \mathrm{mL}$ compared to the most active antibiotic monotherapy) whereas imipenem/trimethoprim-sulfamethoxazole combination regimen showed synergy against one strain (less $3.32 \log _{10} \mathrm{CFU} / \mathrm{mL}$ ). No antagonist effect or major re-growth was observed in all combination regimens, whereas 11 out of 12 of $\beta$-lactam monotherapy regimens were associated with re-growth of bacteria. However, all $\beta$-lactam monotherapy regimens exhibited rapid and stronger killing activities against BUPS/07/14, in the initial 12 hours compared to $\beta$-lactam/ trimethoprimsulfamethoxazole combination regimens. The combination of $\beta$-lactams with trimethoprimsulfamethoxazole demonstrated better killing effect at 24 hours compared to monotherapy and no major bacterial regrowth was observed. Nevertheless, delay in killing activities of $\beta$-lactam/trimethoprim-sulfamethoxazole combination regimens against BUPS/07/14 need further examination because this phenomenon can lead to treatment failure in some patients.
\end{abstract}

Keywords: Melioidosis; Burkholderia pseudomallei; $\beta$-lactam antibiotics; trimethoprimsulfamethoxazole; drug combinations.

\section{INTRODUCTION}

Melioidosis has been recognised as the most neglected tropical disease (Currie \& Kaestli, 2016). The disease was traditionally endemic in Southeast Asia and Northern Australia, but it is now spread to the Indian subcontinent, China, Caribbean, Africa and Middle East (Dance, 2000). The host can acquire melioidosis agent, Burkholderia pseudomallei through ingestion, direct inoculation or inhalation (Ong et al., 2016). Melioidosis has a broad range of symptoms and signs which increasethe possibility of misdiagnosis, thus resulting in treatment delay (Deris et al., 2010). In Malaysia, cases of melioidosis are relatively high in hyper-endemic areas especially in states where agriculture is the main economic activity. Recently, melioidosis cases in Kedah and Pahang states have been reported at 16.35 per 100000 populations and 4.3 per 100000 populations per year, respectively (Abu Hassan et al., 2019).

Current recommended regimens for the intensive phase of melioidosis therapy are ceftazidime or carbapenem for at least 10-14 days and followed by the eradication phase using oral trimethoprim-sulfamethoxazole (SXT) or doxycycline (Lipsitz et al., 2010; Ministry of Health, 2014). SXT is recommended to be added to ceftazidime or carbapenem in 
the intensive phase of therapy only in specific clinical presentations with focal infections or abscess (Lipsitz et al., 2010; Dance, 2014; Currie, 2015).

In Malaysia, with this treatment guideline, the mortality rate can be as high as $65 \%$, especially in cases associated with septicaemia (Deris et al., 2010) with more than $50 \%$ of the deaths occurred within 48 hours after hospital admission (Yazid et al., 2017). The mortality rate is particularly high even though with the use of antibiotics combination (Ganesan et al., 2020). The role of combination therapy needs serious attention and deeper investigation to improve the treatment outcome of melioidosis in future. Here, we investigated the bactericidal effects of $\beta$-lactams and SXT combinations against $B$. pseudomallei strains from Malaysia.

\section{METHODOLOGY}

\section{Institutional Approval}

This study has been approved by the Universiti Sains Malaysia Research Ethics Committee (Ref: USM/JEPeM/16110493). All safety trainings and precautions were carried out in accordance with the safety standard ruled by Department of Medical Microbiology and Parasitology, School of Medical Science, Health Campus, Universiti Sains Malaysia while working with $B$. pseudomallei.

\section{Bacterial strains}

Four clinical strains were selected based on the genotypes that are frequently found in Malaysia. BUPS/12/14, BUPS/07/ 14 , BUPS/07/13 and BUPS/91/08 from sequences type 54, 376, 1322 and 1326 of previous study respectively (Zueter et al., 2015). The isolates were kept at $-80^{\circ} \mathrm{C}$ before use.

\section{Determination of minimum inhibitory concentrations (MICs) of antibiotics}

Minimal inhibitory concentrations (MICs) of SXT (Sigma-Aldrich, St. Louis, MO), amoxicillin-clavulanate (GlaxoSmithKline, Middlesex, UK; AMC), ceftazidime (GlaxoSmithKline, Middlesex, UK) and imipenem (Merck Sharp \& Dohme, Kenilworth, NJ) were performed by microdilution broth method using U-bottomed 96-wells plates, according to Clinical and Laboratory Standard Institute (CLSI) guidelines (CLSI, 2015). The stock solution of antibiotics was prepared by diluting $\sim 5.12 \mathrm{mg}$ of antibiotics powder in solvent to obtain a final solution of $5.12 \mathrm{mg} / \mathrm{mL}$. The concentrations of antibiotics used in this study ranged from $0.125 \mathrm{mg} / \mathrm{L}$ to $128 \mathrm{mg} / \mathrm{L}$. The antibiotic solution was two-fold diluted in Mueller Hinton broth (MHB) and $100 \mu \mathrm{L}$ of antibiotic solution was transferred into 96 wells plate accordingly. About 2 to 3 colonies of $B$. pseudomallei were suspended into normal saline solution until value of $0.5 \mathrm{McF}$ arland turbidity was achieved. A 1:100 dilution of bacterial culture was performed by adding $100 \mu \mathrm{L}$ of bacterial suspension into 9.9 $\mathrm{mL}$ Mueller Hinton broth. Then, $100 \mu \mathrm{L}$ of the bacterial suspension from the dilution tube was inoculated into 96-wells plate. The plate was incubated for 24 hours at $37^{\circ} \mathrm{C}$. The MIC of antibiotics was observed by turbidity visualization by unaided eye. MIC is defined as the lowest concentration of the antibiotics showing inhibition of visible growth turbidity (Andrews, 2001).

Based on standard interpretation of MIC from CLSI (2015), the breakpoint of antibiotics used in this study is amoxicillinclavulanate: susceptible $\leq 8 / 4 \mu \mathrm{g} / \mathrm{mL}$; resistance $\geq 32 / 16$ $\mu \mathrm{g} / \mathrm{mL}$, ceftazidime: susceptible $\leq 8 \mu \mathrm{g} / \mathrm{mL}$; resistance $\geq 16$ $\mu \mathrm{g} / \mathrm{mL}$, imipenem: susceptible $\leq 4 \mu \mathrm{g} / \mathrm{mL}$; resistance $\geq 16 \mu \mathrm{g} /$ $\mathrm{mL}$ and trimethoprim-sulphamethoxazole: susceptible $\leq 2 / 38 \mu \mathrm{g} / \mathrm{mL}$; resistance $\geq 4 / 76 \mu \mathrm{g} / \mathrm{mL}$.

\section{Checkerboard method}

Fractional inhibitory concentrations (FICs) of $\beta$-lactam and SXT combinations were examined by broth microdilution checkerboard method in the same manner as the susceptibility tests. The concentrations of antibiotics were reduced to four or five of two-fold dilution below the MICs. The combination was considered as synergy when the fractional inhibitory concentration index $(\Sigma F I C)$ was equal to or less than 0.5 and antagonism when the $\Sigma$ FIC was greater than 4 . Indifference was indicated by $\Sigma$ FIC value more than 0.5 or equal to or less than 4 (White et al., 1996).

\section{Time-kill studies}

The bactericidal activity was examined by $24 \mathrm{~h}$ static timekill using $1 \times$ MIC of each antibiotic. The mid-log phase bacterial suspension of $1 \times 10^{6} \mathrm{CFU} / \mathrm{mL}$ was used as initial inoculums. All tubes containing bacterial suspension were incubated at $37^{\circ} \mathrm{C}$ in an incubator, shaking at $150 \mathrm{rpm}$. Quantitative culture was performed by serial dilution and spread on nutrient agar plates at time intervals of $0,3,6,12$ and 24 hours. The plates were then incubated at $37^{\circ} \mathrm{C}$ for 18-24 hours for colony count.

Synergy was defined as a reduction of viable colonies by $2-\log _{10}$ of the most active single antibiotic in the regimen at 24 hours as well as a decrease by $2-\log _{10}$ compared to initial inoculums. Indifference was defined as a reduction of viable colonies by $1-\log _{10}$ whereas antagonism was defined as an increase of viable colonies by $2-\log _{10}$ of the interaction at 24h (White et al., 1996). Bacteriostatic and bactericidal activities were defined as $<3-\log _{10}$ and $\geq 3-\log _{10}$ $\mathrm{CFU} / \mathrm{mL}$ reductions in 24 hours, respectively, in relative to the initial inoculums (CLSI, 2015; Smith et al., 2018).

\section{RESULTS}

The MICs of all tested antibiotics against all four strains were within the susceptibility range of CLSI breakpoints except for SXT against BUPS/07/14, which showed a MIC of $4 \mu \mathrm{g} / \mathrm{mL}$ (the loweest breakpoint for resistance) (Table 1).

The $\Sigma$ FIC values of the $\beta$-lactam/SXT combination regimens for all four strains indicated indifference activity with values ranging from 0.75 to 4.00 (Table 2).

Table 1. Bacterial strains used in this study and their minimum inhibitory concentrations

\begin{tabular}{|c|c|c|c|c|c|}
\hline \multirow{2}{*}{ Bacterial strains } & \multirow{2}{*}{ Sequence type* } & \multicolumn{4}{|c|}{ Minimum inhibitory concentrations $(\mu \mathrm{g} / \mathrm{mL}) \#$} \\
\hline & & SXT & AMC & CAZ & IMP \\
\hline BUPS/12/14 & 54 & 2 & 4 & 2 & 0.5 \\
\hline BUPS/07/14 & 376 & 4 & 8 & 4 & 0.5 \\
\hline BUPS/07/13 & 1322 & 1 & 8 & 2 & 0.5 \\
\hline BUPS/91/08 & 1326 & 0.5 & 8 & 2 & 1 \\
\hline
\end{tabular}

\# Trimethoprim-sulfamethoxazole (SXT), imipenem (IMP), ceftazidime (CAZ), amoxicillin-clavulanate (AMC).

*Sequence type were based on our previous study (Zueter et al., 2015). 
In the single antibiotic regimen, the bactericidal activities ( $>3-\log _{10}$ reduction form initial inoculum) were observed in five out of sixteen regimens i.e. imipenem against BUPS/12/14, BUPS/07/13 and BUPS/91/08 and SXT against BUPS/07/13 and BUPS/91/08 (Table 3). Nevertheless, reductions of growth were observed at various time points in all single $\beta$-lactam regimens but regrowth occurred after 12 hours of interaction in eleven out of twelve regimens (Figure 1). Six of these regrowth at 24 hours were more than initial inoculum. Imipenem was the only single $\beta$-lactam antibiotic regimen not associated with regrowth against BUPS/12/14 [Figure 1(A)]. Whereas, three out of four SXT single antibiotic regimens were not associated with regrowth. The reduction was less prominent SXT single antibiotic regimen against BUPS/07/14 and regrowth occurred in BUPS/12/14.

In combination regimens, the bactericidal activities were documented in eight out of twelve regimens. All combination regimens were associated with viable bacterial count at 24 hours lower than initial inoculum. The synergy effects at 24 hours were observed in ceftazidime/SXT against BUPS/07/13, BUPS/07/14 and BUPS/91/08, and imipenem/SXT against BUPS/ $07 / 14$ (Table 3 ). There was no major regrowth observed in combination regimens against these strains. However, there were few small regrowth at various time points; 6 hours of imipenem/SXT against BUPS/07/14, 12 hours of imipenem/ SXT against BUPS/07/13 and BUPS/91/08, and 12 hours of ceftazidime/SXT and amoxicillin-clavulanate/SXT against BUPS/07/13 (Figure 1).

In this study, we found rapid and stronger killing activities against BUPS/07/14 in the early hours $(3,6$ and 12 hours) for all $\beta$-lactams monotherapy regimens compared to their SXT combination regimens [Figure 1 (C)]. The slow killing effects of the combination regimes were also observed in AMC/SXT and ceftazidime/SXT regimens against BUPS/12/14, in which the bacterial killing activity was only observed after 12 hours of incubation compared to 6 and 12 hours in their single antibiotic regimens. The imipenem/SXT combination against these strains had similar pattern with the imipenem single antibiotic regimen [Figure $1(A)$ ].

\section{DISCUSSION}

Antibiotic combination regimen is one of the strategies to improve the treatment efficacy and thus, reduce the mortality rate of infections by resistant organisms. Inhibition of different targets has been used in treating Mycobacterium tuberculosis infections (Worthington \& Melander, 2013). While $B$. pseudomallei is similar to $M$. tuberculosis in term of

Table 2. $\Sigma$ FIC index values of checkerboard assay and interpretation of activity of trimethoprim-sulfamethoxazole and its $\beta$-lactam combinations

\begin{tabular}{|c|c|c|c|}
\hline $\begin{array}{l}\text { Combinations } \\
\text { of antibiotics }\end{array}$ & Strains & $\Sigma$ FIC values & Interpretations \\
\hline \multirow[t]{4}{*}{ SXT + AMC } & BUPS/12/14 & 2.50 & Indifference \\
\hline & BUPS/07/13 & 1.00 & Indifference \\
\hline & BUPS/07/14 & 2.50 & Indifference \\
\hline & BUPS/91/08 & 0.75 & Indifference \\
\hline \multirow[t]{4}{*}{$S X T+C A Z$} & BUPS/12/14 & 4.00 & Indifference \\
\hline & BUPS/07/13 & 1.00 & Indifference \\
\hline & BUPS/07/14 & 1.00 & Indifference \\
\hline & BUPS/91/08 & 1.00 & Indifference \\
\hline \multirow[t]{4}{*}{$S X T+I M P$} & BUPS/12/14 & 1.00 & Indifference \\
\hline & BUPS/07/13 & 0.98 & Indifference \\
\hline & BUPS/07/14 & 1.00 & Indifference \\
\hline & BUPS/91/08 & 0.98 & Indifference \\
\hline
\end{tabular}

\# Trimethoprim-sulfamethoxazole (SXT), imipenem (IMP), ceftazidime (CAZ), amoxicillin-clavulanate (AMC).

Synergy - $\Sigma \mathrm{FIC} \leq 0.5$; Antagonism - $\Sigma \mathrm{FIC}>4$; Indifference $-\Sigma \mathrm{FIC}>0.5$ and $\leq 4$.

Table 3. Interpretation of time-kill curve of $\beta$-lactam/trimethoprim-sulfamethoxazole combinations against four clinical strains of $B$. pseudomallei at $24 \mathrm{~h}$

\begin{tabular}{|c|c|c|c|c|c|}
\hline Antibiotics $\#$ & $\begin{array}{l}\text { Killing activity* } \\
\left(\log _{10} \mathrm{CFU} / \mathrm{mL}\right)\end{array}$ & $\begin{array}{l}\text { Combinations of } \\
\text { antibiotics }^{\#}\end{array}$ & $\begin{array}{l}\text { Killing activity* } \\
\left(\log _{10} \mathrm{CFU} / \mathrm{mL}\right)\end{array}$ & $\begin{array}{l}\text { Interaction at } 24 \mathrm{~h}^{* *} \\
\left(\log _{10} \mathrm{CFU} / \mathrm{mL}\right)\end{array}$ & Interpretation \\
\hline \multicolumn{6}{|l|}{ BUPS/12/14 } \\
\hline SXT & 0.03 & & & & \\
\hline AMC & 0.96 & $A M C+S X T$ & -1.91 & $-2.01^{a}$ & Indifference \\
\hline CAZ & 0.38 & $\mathrm{CAZ}+\mathrm{SXT}$ & -0.55 & -0.66 & Indifference \\
\hline IMP & -4.79 & $\mathrm{IMP}+\mathrm{SXT}$ & -4.51 & 0.40 & Indifference \\
\hline \multicolumn{6}{|l|}{ BUPS/07/13 } \\
\hline SXT & -3.48 & & & & \\
\hline AMC & 1.64 & $A M C+S X T$ & -5.60 & -1.95 & Indifference \\
\hline CAZ & -0.25 & $\mathrm{CAZ}+\mathrm{SXT}$ & -5.92 & -2.25 & Synergy \\
\hline IMP & -3.55 & $\mathrm{IMP}+\mathrm{SXT}$ & -5.15 & -1.64 & Indifference \\
\hline \multicolumn{6}{|l|}{ BUPS/07/14 } \\
\hline SXT & -0.05 & & & & \\
\hline AMC & 1.44 & $\mathrm{AMC}+\mathrm{SXT}$ & -1.97 & -1.54 & Indifference \\
\hline CAZ & -0.14 & $\mathrm{CAZ}+\mathrm{SXT}$ & -2.46 & -2.41 & synergy \\
\hline IMP & 0.15 & $\mathrm{IMP}+\mathrm{SXT}$ & -3.28 & -3.32 & synergy \\
\hline \multicolumn{6}{|l|}{ BUPS/91/08 } \\
\hline SXT & -2.20 & & & & \\
\hline AMC & 1.36 & $A M C+S X T$ & -3.38 & -1.29 & Indifference \\
\hline CAZ & -0.22 & $\mathrm{CAZ}+\mathrm{SXT}$ & -4.41 & -2.38 & Synergy \\
\hline IMP & -4.45 & $\mathrm{IMP}+\mathrm{SXT}$ & -6.00 & -1.32 & Indifference \\
\hline
\end{tabular}

\# Trimethoprim-sulfamethoxazole (SXT), amoxicillin-clavulanate (AMC), ceftazidime (CAZ), imipenem (IMP).

* Killing activity- $\log _{10} \mathrm{CFU} / \mathrm{mL}$ differences between at initial inoculums and at $24 \mathrm{~h}$.

** Synergy $\geq 2-\log _{10}$ reduction, indifference $\pm<2-\log _{10}$, antagonism $\geq 2-\log _{10}$ increase.

a Although the combination $\geq 2-\log 10$, the killing activity of $A M C+S X T$ is $<2-\log _{10} C F U / m L$, so did not fulfill the criteria of synergy. 


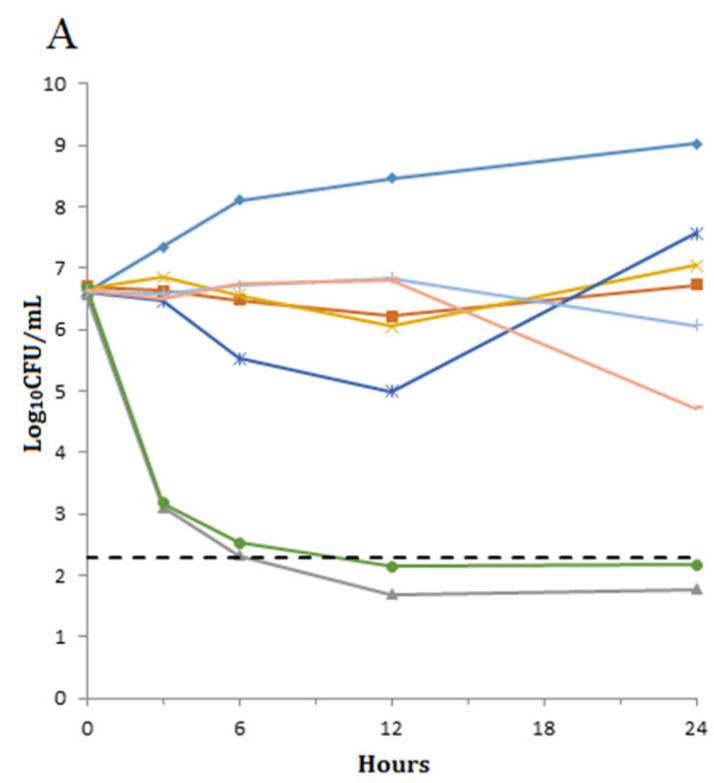

\section{$\mathrm{B}$}
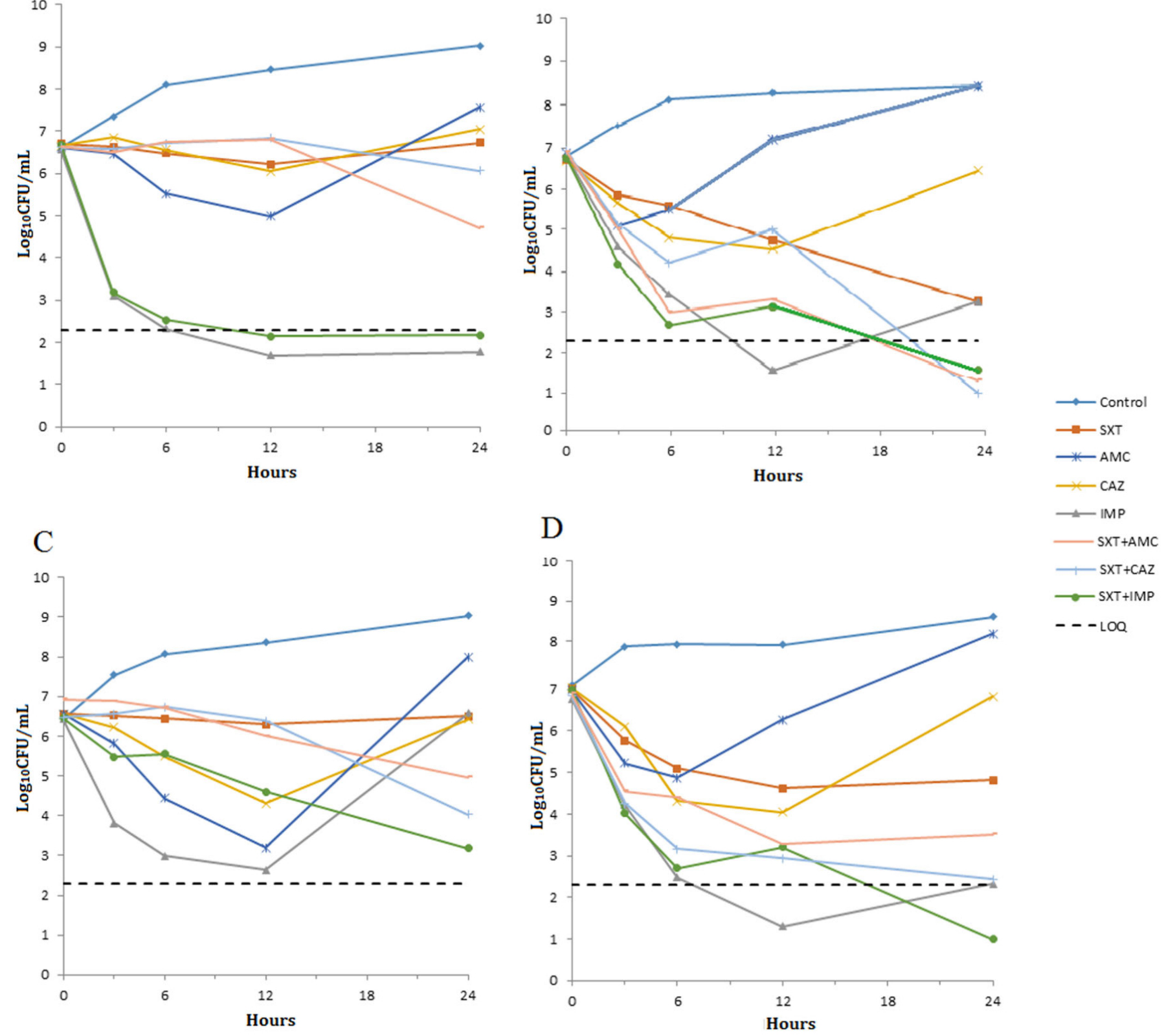

Figure 1. Time-kill curve of antibiotics in combination with trimethoprim-sulfamethoxazole against strain BUPS/12/14 (A); BUPS/ 07/13 (B); BUPS/07/14 (C) and BUPS/91/08 (D). Trimethoprim-sulfamethoxazole (SXT), amoxicillin-clavulanate (AMC), ceftazidime (CAZ) and imipenem (IMP). The dash horizontal line indicates limit of quantification (LOQ). This figure appears in colour in the online version of JAC and in black and white in the print version of JAC.

intrinsically resistant to many antibiotics, at this moment, no such recommendation available in treating melioidosis except in focal infections (Lipsitz et al., 2010; Dance, 2014). SXT has been recommended to be used in combination with ceftazidime or carbapenem because of excellent tissue penetration (Dance, 2014; Currie, 2015). SXT is also being used to treat other intracellular pathogens due to having different activity site from $\beta$-lactams (Zinner \& Mayer, 2015). Combination of two antibiotics with different mechanisms of action is expected to enhance the bacterial killing activity because when the bacteria started to become resistant to one antibiotic, the other antibiotic is supposed to inhibit the bacterial infection successfully (Ankomah et al., 2013). Furthermore, SXT is active against B. pseudomallei and being used as monotherapy in the maintenance phase of the melioidosis therapy (Currie, 2015). With all these arguments, although there is a lack of clinical evidence to support the combination (Dance, 2014; Currie, 2015), SXT is worth to be tested again in vitro as a potential antibiotic to be used in combination with $\beta$-lactams in the intensive phase of the melioidosis therapy.

Compared to our previous study that showed no additional benefit of adding other active antibiotics against B. pseudomallei such as, doxycycline to $\beta$-lactams (Mohamad et al., 2018), in this study we found that the ceftazidime/SXT combination demonstrated synergy against three out of four tested strains whereas the imipenem/SXT combination regimen showed synergy against one out of four strains. There was no antagonist effect of the $\beta$-lactam/SXT combinations in checkerboard as well as in time-kill analysis. Furthermore, there was no major re-growth in the combination regimens compared to $\beta$-lactam monotherapy 
regimens, where eleven out of twelve experiments were associated with re-growth of bacteria.

All these evidences in line with the use of the $\beta$-lactam/ SXT combinations in the treatment of melioidosis. However we found rapid and stronger killing activities in early hours of all $\beta$-lactams monotherapy regimens, compared to their SXT combination regimens against BUPS/07/14. The similar trend was observed when using doxycycline as second antibiotic. Adding doxycycline to $\beta$-lactams regimens led to attenuation and delay in the bacterial killing activity against three out of four tested strains. This is particularly prominent on the imipenem monotherapy at 3, 6 and 12 hours, compared to the doxycycline/imipenem combination against BUPS/12/ 14, BUPS/07/14 and BUPS/91/08. AMC and ceftazidime monotherapies were also superior than their doxycycline combination regimens at 3, 6 and 12 hours against BUPS/07/ 14 and BUPS/91/08 (Mohamad et al., 2018).

We need to further evaluate this phenomenon in order to advice the use of combination therapy in the clinical practice. This is probably due to the activity of $\beta$-lactam antibiotics which are mainly on actively dividing cells, on the other hand, the inhibition of growth induced by SXT should result in an overall reduction of actively dividing cells (Ocampo et al., 2014). The secondary resistance of the same class of antibiotics with same mode of action is common and may lead to the resistance mechanism of different class of antibiotics (Zamani et al., 2020). This resulted in reduce efficacy especially during early part of the experiments when the $\beta$-lactam/ SXT combination was used from the beginning of the therapy. Therefore, the time of commencement of the second antibiotic probably play an important role in the bactericidal activity of the combinations.

In conclusion, this study has shown the benefits of the $\beta$-lactam/SXT combinations over the monotherapy against a few strains of $B$. pseudomallei from Malaysia. However, we also found attenuation and delay in the bactericidal activity of the combination regimens against some strains, which may lead to the treatment failure. Further study is warranted to understand this phenomenon in order to increase the efficacy of combination therapy against melioidosis. Furthermore, pharmacodynamic examination to find an optimum time to initiate the second antibiotic is also critical to improve patient's survival.

\section{ACKNOWLEDGEMENT}

The authors express their gratitude to the staffs at the Medical Microbiology and Parasitology laboratory and Hospital Universiti Sains Malaysia for their help and services throughout this study. This study was supported by USM short term grant (304.PPSP.61313057).

\section{Conflict of interest statement}

The authors declare that they have no competing interests.

\section{REFERENCES}

Abu Hassan, M.R., Aziz, N., Shafie, Z., Mayala, B., Donohue, R.E., Pani, S.P. \& Michael, E. (2019). Socio-epidemiological and land cover risk factors for melioidosis in Kedah, Northern Malaysia. PLoS Neglected Tropical Diseases 13: e0007243. https://doi.org/10.1371/journal.pntd.0007243

Andrew, J.M. (2001). Determination of minimum inhibitory concentrations. The Journal of Antimicrobial Chemotherapy 48: 5-16. https://doi.org/10.1093/jac/48.suppl_1.5
Ankomah, P., Johnson, P.J.T. \& Levin, B.R. (2013). The pharmaco-, population and evolutionary dynamics of multi-drug therapy: experiments with $S$. aureus and E. coli and computer simulations. PLoS Pathogens 9: e1003300. https://doi.org/10.1371/journal.ppat.1003300

Clinical and Laboratory Standards Institute (CLSI) (2015). M45 Methods for Antimicrobial Dilution and Disk Susceptibility Testing of Infrequently Isolated or Fastidious Bacteria $3^{\text {rd }}$ edition. Wayne: CLSI, pp. 60-63. https://goums.ac.ir/files/deputy_treat/md_labs_ef39a/ files/CLSI-M45ed3e-2018(1).pdf. Accessed 3 February 2015.

Currie, B.J. (2015). Melioidosis: evolving concepts in epidemiology, pathogenesis, and treatment. Seminars in Respiratory and Critical Care Medicine 36: 111-125. https:// doi.org/10.1055/s-0034-1398389

Currie, B.J. \& Kaestli, M. (2016). A global picture of melioidosis. The Nature 529: 290-291. https://doi.org/ $10.1038 / 529290 a$

Dance, D. (2014). Treatment and prophylaxis of melioidosis. International Journal of Antimicrobial Agents 43: 310-318. https://doi.org/10.1016/j.ijantimicag.2014.01.005

Dance, D.A.B. (2000). Melioidosis as an emerging global problem. Acta Tropica 94: 115-119. https://doi.org/10.1016/ s0001-706x(99)00059-5

Deris, Z.Z., Hasan, H. \& Mohd Noor, S.S. (2010). Clinical characteristics and outcomes of bacteraemic melioidosis in a teaching hospital in a norheastern state of Malaysia: a five-year review. Journal of Infection in Developing Countries 4: 430-435. https://doi.org/10.3855/jidc.491

Ganesan, H., Embi, N. \& Hasidah, M.S. (2020). Potential of repurposing chloroquine as an adjunct therapy for melioidosis based on a murine model of Burkholderia pseudomallei infection. Tropical Biomedicine 37: 303-317.

Lipsitz, R., Garges, S., Aurigemma, R., Beccam, P., Blaney, D.D., Cheng, A.C., Currie, B.J., Dance, D., Gee, J.E., Larsen, J. et al. (2012). Workshop on treatment of and postexposure prophylaxis for Burkholderia pseudomallei and B. mallei infection 2010. Emerging infectious diseases 18: e2. https:// doi.org/10.3201/eid1812.120638

Ministry of Health Malaysia (2014). National Antibiotic Guideline $2^{\text {nd }}$ edition. Petaling Jaya: Ministry of Health Malaysia, pp. 158-159. https://www.pharmacy.gov.my/v2/ sites/default/files/document-upload/nationalantibiotic-guideline-2014-full-versionjun2015_1.pdf. Accessed 27 December 2016.

Mohamad, N.I., Harun, A., Hasan, H. \& Deris, Z.Z. (2018). InVitro activity of doxycycline and beta-lactam combinations against different strains of Burkholderia pseudomallei. Indian Journal of Microbiology 58: 244-247. https://doi.org/10.1007/ s12088-018-0722-4

Ocampo, P.S., Lázár, V., Papp, B., Arnoldini, M., Wiesh, P.A., Busa-Fekete, R., Fekete, G., Pal, C., Ackermann, M. \& Bonhoeffer, S. (2014). Antagonism between bacteriostatic and bactericidal antibiotics is prevalent. Antimicrobial Agents and Chemotherapy 58: 4573-4582. https://doi.org/ 10.1128/AAC.02463-14

Ong, G.H., Choh, L.C., Vellasamy, K.M., Mariappan, V., Chang, L.Y. \& Vadivelu, J. (2016). Experimental phage therapy for Burkholderia pseudomallei infection. PLOS ONE 11: e0158213. https://doi.org/10.1371/journal.pone.0158213

Smith, J.R., Juwon, Y., Rice, S., Stamper, K., Kebriael, R. \& Rybak, M.J. (2018). Combination of tedizolid and daptomycin against methicillin-resistant. Antimicrobial Agents and Chemotherapy 62: e00101-18. https://doi.org/10.1128/ AAC.00101-18 
White, R.L., Burgess, D.S., Manduru, M. \& Bosso, J.A. (1996). Comparison of three different in vitro methods of detecting synergy: time-kill, checkerboard, and E test. Antimicrobial Agents and Chemotherapy 40: 1914-1918. https://doi.org/ 10.1128/AAC.40.8.1914

Worthington, R.J. \& Melander, C. (2013). Combination approaches to combat multidrug-resistant bacteria. Trends in Biotechnology 31: 177-184. https://doi.org/10.1016/ j.tibtech.2012.12.006

Yazid, M.B., Fauzi, M.H, Hasan, H., Md Noh, A.Y. \& Deris, Z.Z. (2017). An 11-Year Analysis of Emergency Presentations of Melioidosis in Northeastern Malaysia. Journal of Immigrant and Minority Health 19: 774-777. https://doi.org/10.1007/ s10903-016-0429-8
Zamani, A., Zueter, A.R., Muhd Besari, A., Harun, A., Hasan, H. \& Deris, Z.Z. (2020). Reduced susceptibility of Burkholderia pseudomallei following exposure to carbapenem. Tropical Biomedicine 37: 783-790. https://doi.org/10.47665/tb.37.3.783

Zinner, S.H. \& Mayer, K.H. (2015). Mandell, Douglas, and Bennett's Principles and Practice of Infectious Diseases. $8^{\text {th }}$ edition. Philadelphia: Elsevier Saunders, pp. 475-486.

Zueter, A.M., Abdul Rahman, Z., Chan, Y.Y. \& Harun, A. (2015). Brief communication genotyping of Burkholderia pseudomallei revealed high genetic variability among isolates from a single population group. International Journal of Molecular Epidemiology and Genetics 6: 41-47. 